\title{
Nomogram for the prediction of individualized overall survival of patients diagnosed with small cell esophageal carcinoma
}

\author{
Di Liu ${ }^{1,2,3 \#}$, Junmiao Wen ${ }^{1,2,3}$, Jiayan Chen ${ }^{1,2,3}$, Min Fan $^{1,2,3 *}$, Zhen Zhang ${ }^{1,2,3 *}$ \\ ${ }^{1}$ Department of Radiation Oncology, Fudan University Shanghai Cancer Center, Shanghai, China; ${ }^{2}$ Department of Oncology, Shanghai Medical \\ College, Fudan University, Shanghai, China; ${ }^{3}$ Shanghai Key Laboratory of Radiation Oncology, Shanghai, China \\ Contributions: (I) Conception and design: D Liu, Z Zhang, M Fan; (II) Administrative support: Z Zhang, M Fan; (III) Provision of study materials or \\ patients: J Chen, M Fan; (IV) Collection and assembly of data: D Liu, J Wen; (V) Data analysis and interpretation: D Liu, J Wen; (VI) Manuscript \\ writing: All authors; (VII) Final approval of manuscript: All authors. \\ \#These authors contributed equally to this work. \\ *These authors are corresponding authors and contributed equally to this work. \\ Correspondence to: Min Fan, MD; Zhen Zhang, MD. Department of Radiation Oncology, Fudan University Shanghai Cancer Center, Shanghai, \\ China; Department of Oncology, Shanghai Medical College, Fudan University, Shanghai 200032, China; Shanghai Key Laboratory of Radiation \\ Oncology, Shanghai, China. Email: minfan20141212@hotmail.com; zhen_zhang@fudan.edu.cn.
}

Background: A nomogram was developed for the estimation of individualized overall survival (OS) of patients diagnosed with small cell esophageal carcinoma (SCEC).

Methods: From the SEER dataset, 427 patients diagnosed with SCEC during the period from 2004 to 2015 were selected as training sets. For the establishment of a nomogram capable of estimating the OS possibility of patients diagnosed with SCEC, a group of independent prognostic factors were identified and incorporated. The effectiveness of the nomogram was then both externally and internally verified among 159 patients from Fudan University Shanghai Cancer Center (FUSCC) who were diagnosed with SCEC between 2006 and 2015. The predictive accuracy and discriminative ability of the nomogram were measured by concordance index (C-index). Comparisons between nomogram and the AJCC staging systems (6th and 7 th) were performed with calibration plots and area under the curves (AUC) values.

Results: We identified age, gender, primary site, SEER stage, surgery, radiotherapy, and chemotherapy as seven independent risk factors which were then used to set up the nomogram. Calibration curves indicated that the prediction of the nomogram was consistent with real observations for the possibilities of 1-, 3-, and 5 -year OS, and applying the nomogram to the cohort for validation led to reproducible results. Moreover, the C-indices and AUC values were higher in the nomogram than those in the AJCC staging system AJCC which is also aimed at the prediction of OS.

Conclusions: This study resulted in the establishment of the first nomogram for the prediction of individualized OS of patients diagnosed with SCEC. The accuracy rate of prediction of this model may be higher than previously established staging systems.

Keywords: Small cell esophageal carcinoma (SCEC); overall survival (OS); prognostic model

Submitted Jul 09, 2021. Accepted for publication Aug 16, 2021.

doi: $10.21037 /$ atm-21-3900

View this article at: https://dx.doi.org/10.21037/atm-21-3900

\section{Introduction}

Neuroendocrine carcinoma (NEC) is an esophageal tumour with low incidence, constituting only $3 \%$ of all esophageal cancers $(1,2)$. Of tumours classified by the WHO originating in the digestive system, NECs are considered as poorly-differentiated carcinomas, whose mitotic count is higher than 20 per 10 high power field, and whose index of proliferation is higher than 20 percent. Both small 
and large cell carcinomas exist in NECs $(3,4)$. Primary small cell esophageal carcinoma (PSCEC) is the most common extrapulmonary small cell carcinomas $(5,6)$ and its prevalence is increasing overtime $(7,8)$. Most patients with SCEC have a poor prognosis, with a median survival time of is only 8-13 months following diagnosis (5).

Due to the rarity and tumour heterogeneity of SCEC, a specific staging system, clinical trials, or standard treatment have not as yet been established. Additionally, as SCEC is a highly aggressive malignancy with dismal survival, considerable controversy regarding its prognostic factors exists, with the few studies investigating this being retrospective, with small sample size, and producing inconsistent results (9-13).

A nomogram is a statistical model based on a set of vital factors and aimed to estimate the possible result of a clinical event, such as the recurrence rate of a disease or the possibility of death (14). It is widely regarded as a tool that can reliably predict the outcomes of clinical treatments of numerous cancers, and in some $(15,16)$, has been verified as producing higher accuracy than previously developed staging systems. While nomograms are now used as alternative tools and are even seen as a novel criterion to manage cancer patients, none have been established to estimate the OS of patients diagnosed with SCEC. Therefore, it is imperative that investigations should be made in which SCEC OS is estimated on the basis of a large-scale cohort of patients.

For a precise estimation of the clinical treatment effect of patients diagnosed with SCEC, a nomogram was set up to investigate the contribution of each clinical variable bearing significance, as well as the relevant SEER data. In addition, an independent group of SCEC patients from Fudan University Shanghai Cancer Center (FUSCC) were employed to externally validate the model. We present the following article in accordance with the TRIPOD reporting checklist (available at https://dx.doi.org/10.21037/atm-21-3900).

\section{Methods}

\section{Study population}

This retrospective research was approved by the Institutional Review Board of FUSCC. The study was conducted in accordance with the Declaration of Helsinki (as revised in 2013). With the support of the National Cancer Institute, information was sourced and obtained from 18 cancer registries in the SEER dataset which cover around one third of the total population of the United States. By referring to the 2008 ICD-0-3/WHO between 2004 and 2015, all patients diagnosed with SCEC were selected. Several covariates were also investigated incorporating race, gender, age when the patient was diagnosed, primary tumour location, registration region, marital status, SEER summary stage, tumour-nodemetastasis (TNM) stage (6th and 7th edition), surgery type, and the use of chemotherapy and radiation. On the other hand, SCEC patients treated in the FUSCC between 2006 and 2015 were also selected as a validation cohort, and only those diagnosed with SCEC in the FUSCC for the first time were chosen, while those treated with anti-cancer therapies such as radiotherapy and chemotherapy were not considered. In addition to the aforementioned variables, performance status score (PS), smoking status, alcohol use, food habit, vascular invasion, perineural involvement, and pathology (pure or mixed SCEC) were also sought and included, while patients whose information was incomplete were excluded.

\section{Statistical analyses}

Statistical analysis was performed using R-3.5.2 for Windows. OS was defined as the duration from the time when a patient was initially diagnosed with SCEC to the time when they died or attended their last follow-up, and was the primary endpoint of the research. Continuous data were analyzed using Student's $t$ test and categorical data using Chi-square test. Log-rank tests and Kaplan-Meier survival curves were employed to compare OS between different subgroups of SCEC patients and to delineate features influencing OS. Univariate and multivariable Cox regression analyses were applied for survival analysis. Using the methods in our previous study, a novel nomogram was built based on prognostic parameters derived from the SEER data (17). To evaluate whether the survival predicted by the nomogram was consistent with the observed survival, we referred to its calibration curves, which were validated by 1,000 resamples. We investigated the prognostic performance of the nonogram by using both calibration plots and time-dependent receiver operating characteristic (tdROC) curve analyses, and further compared its performance with that of the AJCC staging system through calculating $\mathrm{C}$-indices and area under the curves (AUC) values. A two-sided $\mathrm{P}$ value smaller than 0.05 suggested statistical significance.

\section{Results}

\section{Patient baseline characteristics}

We retrospectively enrolled 427 patients diagnosed with SCEC in the SEER database between 2004 and 2015. To 
verify the effectiveness of the nomogram, 159 patients were gathered as a cohort from the FUSCC through referring to the set inclusion and exclusion standards, and their baseline clinical pathologic features are summarized in Table 1. Most patients, both in the training group and in the verification group, were male, married, had lymph node metastasis, and received chemotherapy. Lower thoracic SCEC was the most common primary tumor site (44\%) in the SEER database, while middle thoracic SCEC (64.8\%) was the most common site in the SEER database. The SEER cohort were relatively older (mean $67.4 \pm 11.4$ years-of-age) at diagnosis than the validation cohort (mean 61.0 \pm 7.6 years-of-age), were associated with higher probability of distant metastasis (49.4\%), and were more likely to have undergone radiotherapy $(52.5 \%)$. By contrast in the validation cohort, as a regional stage predominated $(45.3 \%)$, most patients received surgery $(60.4 \%)$, while radiotherapy only involved $40.9 \%$ of patients. As listed in Table 1, we retrospectively collected information on PS, pathology (pure or mixed SCEC), smoking status, alcohol use, food habits, vascular invasion, perineural involvement, and surgical type in the validation group.

\section{Independent prognostic factors in the training cobort}

A univariate analysis was performed in the training cohort to clarify the clinical parameters having vital linkages with the OS of SCEC patients. Figure $1 A$ shows that race, gender, age, marital status, CHSDA region, primary tumour site, surgery, SEER stage, and clinical therapies such as chemotherapy and radiotherapy display remarkable associations with OS. However, multivariate analysis showed that race, marital status, and CHSDA region lost their vital roles, while the remaining variables remained significant (Figure 1B). The 3-year OS rates for patients with SEER localized, regional, and distant stages were $27.7 \%$, 20.5\%, and $2.4 \%$, respectively. The Kaplan-Meier survival curves for different SEER stages are shown in Figure $2 A$. Of note, neither the 6th or 7th AJCC TNM staging system was an independent prognostic factor in the Cox regression model, and the Kaplan-Meier survival curves were overlapped among AJCC stages I-IV (Figure 2B,2C). Better survival was found among the patients treated with chemotherapy than those never receiving it, and radiotherapy and surgery were also found to be associated with favourable outcomes.

\section{Prognostic nomogram for OS}

A nomogram incorporating all variables of significance identified by using the covariates mentioned above was developed, and as shown in Figure 3, the nomogram predicting 1-, 3-, and 5-year OS was built on the basis of chosen parameters that had a hazard ratio. The nonogram showed the greatest contributing factor to prognosis was the SEER distant stage, followed by chemotherapy, unknown SEER stage and regional stage, radiotherapy, upper primary tumour site, and male gender. Table S1 shows the exact values of all predictive parameters of the nomogram. Through adding all these values and placing them into the total-point scale, the survival probabilities were calculated.

\section{Validation of the predictive accuracy of the nomogram for OS}

The C-index of the nonogram, which was employed to indicate OS, was 0.71 (95\% CI, 0.67-0.75) in the training set by using $1,000 \times$ bootstrap resampling. The predicted OS was further verified externally in the separate group of 159 patients from the FUSCC, who had been diagnosed with SCEC. The calibration plots presented an optimal agreement between the outcomes predicted by the nomogram and those actually observed in terms of the 1-, 3 -, and 5-year OS (Figure 4A-4C). Internal and external validation results both suggested appreciable reliability of the nomogram.

\section{Comparison of the nomogram with AfCC staging systems}

We did comparisons between the SCEC nomogram with the 6th and 7th AJCC TNM staging systems by calculating $\mathrm{C}$-indices and area under the curves (AUC) values to access the prediction accuracy. The nomogram yielded a significantly larger $\mathrm{C}$-index for predicting $\mathrm{OS}$ in the group aimed at verification compared with that of the AJCC stages [nomogram: 0.71 (95\% CI, 0.67-0.75); TNM 6th: 0.63 (95\% CI, 0.59-0.67); TNM 7th: 0.64 (95\% CI, 0.60-0.68); $\mathrm{P}<0.001]$. Figure $4 D-4 F$ and Table $\mathrm{S} 2$ show the AUC value of the nonogram was slightly higher than that of the TNM staging system (nomogram $v s$. TNM 6th $v s$. 7th, 1-year OS rate, 0.92 vs. 0.59 vs. $0.81 ; 3$-year OS rate, 0.89 vs. 0.67 vs. $0.65 ; 5$-year OS rate, 0.83 vs. 0.61 vs. 0.66 ). These results implied both the short-term OS and the long-term OS were predicted more accurately by the nomogram than the AJCC staging systems.

\section{Discussion}

SCEC is an exceptionally rare diagnosis, with its prognostic 
Table 1 Basic demographic and patient characteristics in the training and validation cohort of SCEC patients

\begin{tabular}{|c|c|c|c|}
\hline Variables & $\begin{array}{l}\text { FUSCC } \\
(n=159)\end{array}$ & $\begin{array}{c}\text { SEER } \\
(\mathrm{n}=427)\end{array}$ & $P$ \\
\hline Race (\%) & & & $<0.001$ \\
\hline NR & $159(100.0)$ & $0(0.0)$ & \\
\hline Others & $0(0.0)$ & $94(22.0)$ & \\
\hline White & $0(0.0)$ & $333(78.0)$ & \\
\hline Gender (\%) & & & 0.001 \\
\hline Female & $38(23.9)$ & $168(39.3)$ & \\
\hline Male & $121(76.1)$ & $259(60.7)$ & \\
\hline CHSDA region (\%) & & & $<0.001$ \\
\hline East & $0(0.0)$ & $153(35.8)$ & \\
\hline Northern Plains & $0(0.0)$ & $84(19.7)$ & \\
\hline NR & $159(100.0)$ & $0(0.0)$ & \\
\hline Pacific Coast & $0(0.0)$ & $167(39.1)$ & \\
\hline Southwest & $0(0.0)$ & $23(5.4)$ & \\
\hline Primary site (\%) & & & $<0.001$ \\
\hline Lower & $35(22.0)$ & $188(44.0)$ & \\
\hline Middle & $103(64.8)$ & $105(24.6)$ & \\
\hline Others & $7(4.4)$ & $101(23.7)$ & \\
\hline Upper & $14(8.8)$ & $33(7.7)$ & \\
\hline TNM 7th (\%) & & & $<0.001$ \\
\hline 1 & $2(1.3)$ & $0(0.0)$ & \\
\hline IA & $12(7.5)$ & $5(1.2)$ & \\
\hline IB & $14(8.8)$ & $24(5.6)$ & \\
\hline IIA & $4(2.5)$ & $24(5.6)$ & \\
\hline IIB & $31(19.5)$ & $19(4.4)$ & \\
\hline III & $1(0.6)$ & $0(0.0)$ & \\
\hline IIIA & $17(10.7)$ & $28(6.6)$ & \\
\hline IIIB & $7(4.4)$ & $0(0.0)$ & \\
\hline IIIC & $20(12.6)$ & $21(4.9)$ & \\
\hline IV & $51(32.1)$ & 168 (39.3) & \\
\hline NR & $0(0.0)$ & 138 (32.3) & \\
\hline
\end{tabular}

Table 1 (continued)
Table 1 (continued)

\begin{tabular}{|c|c|c|c|}
\hline Variables & $\begin{array}{l}\text { FUSCC } \\
(n=159)\end{array}$ & $\begin{array}{c}\text { SEER } \\
(n=427)\end{array}$ & $P$ \\
\hline TNM 6th (\%) & & & $<0.001$ \\
\hline 1 & $14(8.8)$ & $28(6.6)$ & \\
\hline$\| \mathrm{A}$ & $18(11.3)$ & $28(6.6)$ & \\
\hline IIB & 37 (23.3) & $14(3.3)$ & \\
\hline III & 37 (23.3) & $46(10.8)$ & \\
\hline IIIA & $1(0.6)$ & $0(0.0)$ & \\
\hline IVA & $8(5.0)$ & $8(1.9)$ & \\
\hline IVB & $44(27.7)$ & $144(33.7)$ & \\
\hline IV NOS & $0(0.0)$ & $20(4.6)$ & \\
\hline NR & $0(0.0)$ & 139 (32.6) & \\
\hline T stage 7th (\%) & & & $<0.001$ \\
\hline $\mathrm{T} 1$ & $8(5.0)$ & $0(0.0)$ & \\
\hline $\mathrm{T} 1 \mathrm{a}$ & $0(0.0)$ & $51(11.9)$ & \\
\hline $\mathrm{T} 1 \mathrm{~b}$ & $23(14.5)$ & $8(1.9)$ & \\
\hline $\mathrm{T} 2$ & $50(31.4)$ & $41(9.6)$ & \\
\hline $\mathrm{T} 3$ & $51(32.1)$ & $50(11.7)$ & \\
\hline $\mathrm{T} 4$ & $3(1.9)$ & $0(0.0)$ & \\
\hline $\mathrm{T} 4 \mathrm{a}$ & $10(6.3)$ & $38(8.9)$ & \\
\hline $\mathrm{T} 4 \mathrm{~b}$ & $11(6.9)$ & $16(3.7)$ & \\
\hline TX & $3(1.9)$ & $223(52.2)$ & \\
\hline N stage 7th (\%) & & & $<0.001$ \\
\hline No & $38(23.9)$ & $114(26.7)$ & \\
\hline $\mathrm{N} 1$ & $58(36.5)$ & $133(31.1)$ & \\
\hline N2 & $44(27.7)$ & $7(1.6)$ & \\
\hline N3 & $19(11.9)$ & $0(0.0)$ & \\
\hline NX & $0(0.0)$ & $173(40.5)$ & \\
\hline M stage 7 th (\%) & & & $<0.001$ \\
\hline MO & $108(67.9)$ & $143(33.5)$ & \\
\hline M1 & $51(32.1)$ & 168 (39.3) & \\
\hline$M X$ & $0(0.0)$ & $116(27.2)$ & \\
\hline T stage 6th (\%) & & & $<0.001$ \\
\hline $\mathrm{T} 1$ & $31(19.5)$ & $59(13.8)$ & \\
\hline $\mathrm{T} 2$ & $50(31.4)$ & $41(9.6)$ & \\
\hline $\mathrm{T} 3$ & $51(32.1)$ & $50(11.7)$ & \\
\hline $\mathrm{T} 4$ & $24(15.1)$ & $54(12.6)$ & \\
\hline TX & $3(1.9)$ & $223(52.2)$ & \\
\hline
\end{tabular}

Table 1 (continued) 
Table 1 (continued)

\begin{tabular}{|c|c|c|c|}
\hline Variables & $\begin{array}{l}\text { FUSCC } \\
(n=159)\end{array}$ & $\begin{array}{c}\text { SEER } \\
(n=427)\end{array}$ & $P$ \\
\hline $\mathrm{N}$ stage 6th (\%) & & & $<0.001$ \\
\hline NO & $40(25.2)$ & $117(27.4)$ & \\
\hline $\mathrm{N} 1$ & $119(74.8)$ & $136(31.9)$ & \\
\hline NX & $0(0.0)$ & $174(40.7)$ & \\
\hline M stage 6th (\%) & & & $<0.001$ \\
\hline MO & 108 (67.9) & $139(32.6)$ & \\
\hline M1 & $1(0.6)$ & $20(4.7)$ & \\
\hline M1a & $8(5.0)$ & $8(1.9)$ & \\
\hline M1b & $42(26.4)$ & $144(33.7)$ & \\
\hline$M X$ & $0(0.0)$ & $116(27.2)$ & \\
\hline SEER stage (\%) & & & $<0.001$ \\
\hline Localized & 37 (23.3) & 77 (18.0) & \\
\hline Regional & $72(45.3)$ & $76(17.8)$ & \\
\hline Distant & $50(31.4)$ & $211(49.4)$ & \\
\hline Unstaged & $0(0.0)$ & $63(14.8)$ & \\
\hline \multicolumn{4}{|l|}{ VALSG stage } \\
\hline Limited & $115(72.3)$ & NR & \\
\hline Extensive & $44(27.7)$ & NR & \\
\hline Surgery (\%) & & & $<0.001$ \\
\hline No & $63(39.6)$ & 388 (90.9) & \\
\hline Yes & $96(60.4)$ & $39(9.1)$ & \\
\hline Radiotherapy (\%) & & & 0.016 \\
\hline No & $94(59.1)$ & $203(47.5)$ & \\
\hline Yes & $65(40.9)$ & 224 (52.5) & \\
\hline Chemotherapy (\%) & & & 0.006 \\
\hline No & 29 (18.2) & $128(30.0)$ & \\
\hline Yes & $130(81.8)$ & $299(70.0)$ & \\
\hline Age [mean (SD)] & $60.99(7.64)$ & $67.37(11.37)$ & $<0.001$ \\
\hline Marital status (\%) & & & $<0.001$ \\
\hline No & $0(0.0)$ & $192(45.0)$ & \\
\hline Yes & $159(100.0)$ & $235(55.0)$ & \\
\hline Performance status (\%) & & & $<0.001$ \\
\hline 0 & $61(38.4)$ & $0(0.0)$ & \\
\hline 1 & $94(59.1)$ & $0(0.0)$ & \\
\hline 2 & $4(2.5)$ & $0(0.0)$ & \\
\hline NR & $0(0.0)$ & 427 (100.0) & \\
\hline
\end{tabular}

Table 1 (continued)

\begin{tabular}{|c|c|c|c|}
\hline Variables & $\begin{array}{l}\text { FUSCC } \\
(n=159)\end{array}$ & $\begin{array}{c}\text { SEER } \\
(\mathrm{n}=427)\end{array}$ & $P$ \\
\hline \multicolumn{4}{|l|}{ Pathology } \\
\hline Pure SCEC & $131(82.4)$ & NR & \\
\hline Mixed SCEC & $28(17.6)$ & NR & \\
\hline Smoking status (\%) & & & $<0.001$ \\
\hline $\mathrm{N}$ & $68(42.8)$ & $0(0.0)$ & \\
\hline NR & $0(0.0)$ & $427(100.0)$ & \\
\hline $\mathrm{Y}$ & $91(57.2)$ & $0(0.0)$ & \\
\hline Alcohol status (\%) & & & $<0.001$ \\
\hline $\mathrm{N}$ & $91(57.2)$ & $0(0.0)$ & \\
\hline NR & $0(0.0)$ & $427(100.0)$ & \\
\hline Y & $68(42.8)$ & $0(0.0)$ & \\
\hline Food habit (\%) & & & $<0.001$ \\
\hline $\mathrm{N}$ & $76(47.8)$ & $0(0.0)$ & \\
\hline NR & $19(11.9)$ & $427(100.0)$ & \\
\hline $\mathrm{Y}$ & $64(40.3)$ & $0(0.0)$ & \\
\hline Vascular invasion (\%) & & & $<0.001$ \\
\hline NR & $70(44.0)$ & $427(100.0)$ & \\
\hline Y & $36(22.6)$ & $0(0.0)$ & \\
\hline $\mathrm{N}$ & $73(45.9)$ & $0(0.0)$ & \\
\hline Perineural involvement (\%) & & & $<0.001$ \\
\hline NR & $70(44.0)$ & $427(100.0)$ & \\
\hline Y & $16(10.1)$ & $0(0.0)$ & \\
\hline Ivor-Lewis & $62(39.0)$ & $0(0.0)$ & \\
\hline Surgical type (\%) & & & $<0.001$ \\
\hline McKeown & $8(5.0)$ & $0(0.0)$ & \\
\hline No & $70(44.0)$ & $0(0.0)$ & \\
\hline NR & $0(0.0)$ & $427(100.0)$ & \\
\hline Palliative resection & $3(1.9)$ & $0(0.0)$ & \\
\hline Sweet's & $3(1.9)$ & $0(0.0)$ & \\
\hline Three field & $13(8.2)$ & $0(0.0)$ & \\
\hline
\end{tabular}

FUSCC, Fudan University Shanghai Cancer Center; SEER, Surveillance, Epidemiology, and End Results database; NR, not reported; CHSDA, Contract Health Service Delivery Areas; NOS, not of specific; VALSG, Veterans Administration Lung Study Group; SD, standard deviation; SCEC, small cell oesophageal carcinoma.

Table 1 (continued) 

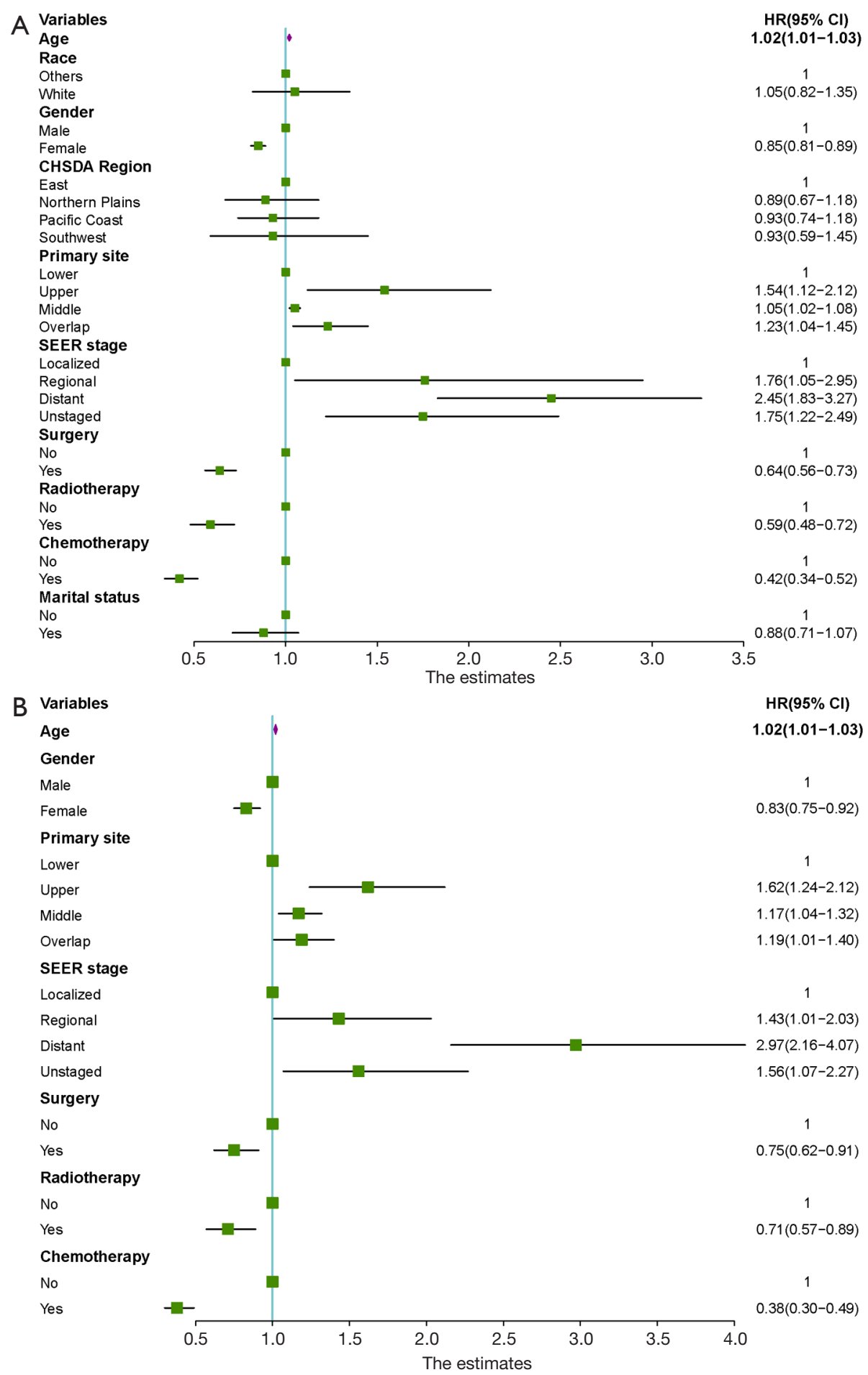

Figure 1 Cox regression analysis for OS in SCEC patients from the training cohort. Univariate (A) and multivariate (B) Cox regression in the SEER cohort. SEER, Surveillance, Epidemiology, and End Results database; SCEC, small cell oesophageal carcinoma. 

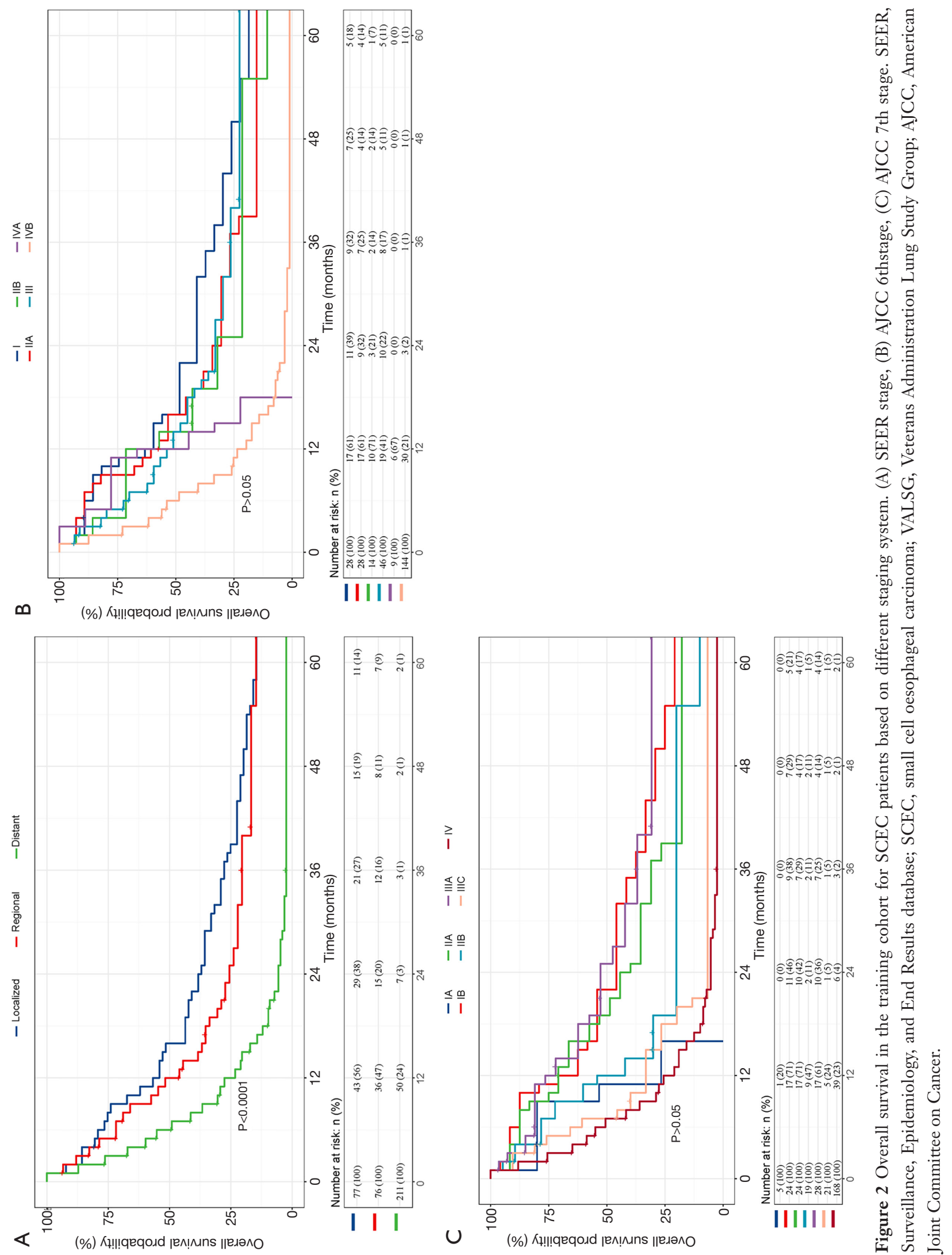

(c) Annals of Translational Medicine. All rights reserved. 


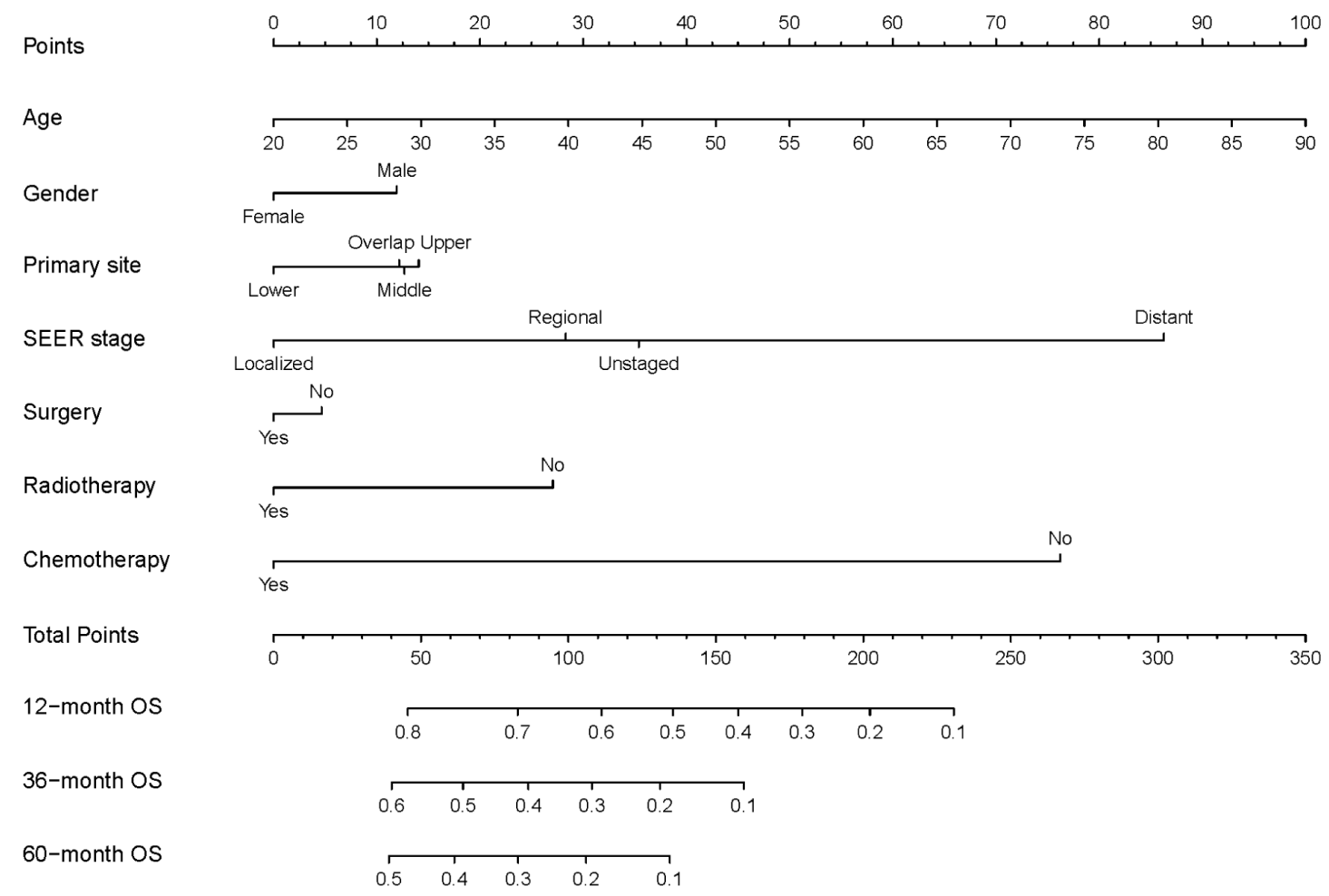

Figure 3 Nomogram estimating 1-, 3-, and 5-year overall survival for SCEC patients based on the SEER cohort. SCEC, small cell oesophageal carcinoma; SEER, Surveillance, Epidemiology, and End Results database.

factors and treatment strategy poorly understood. As existing knowledge on these factors has largely been derived from small series or case reports, there is an urgent need to more accurately determine the survival characteristics of SCEC patients. In this study, the data of many SCEC patients were obtained from the SEER dataset, and a comprehensive nomogram was established for the predictive calculation of their OS. The model showed satisfactory agreement in its application to both training and validation groups of SCEC patients, and obtained superiority over AJCC staging systems, with more accurate individual prediction of survival probability, demonstrating its clinically application. The extremely low incidence of SCEC has led to there being no specific staging system for the disease, and to date, the TNM-based AJCC staging systems have been adopted to assess the survival of patients. However, our results showed the predictive value of the 6th and 7th AJCC staging systems for the estimation of OS in SCEC patients to be poor, and through AJCC stages I$\mathrm{IV}$, the survival curves were obviously overlapped. Instead of TNM stages, SEER stages were incorporated into the multivariate COX analysis and showed none of T, N, or $M$ classifications were significant prognostic factors. The standardization and simplification of SEER staging ensures over-time consistency among various definitions that exert an impact on OS in our nomogram, and accords with previously released statistics (18). However, as there is a wider application of AJCC staging systems in clinical practice, whether TNM stages correlate with the survival of SCEC patients remains controversial. One study of 64 patients suggested that $\mathrm{T}$ stage was an independent prognostic factor (10), with other data suggesting the $\mathrm{N}$ stage was associated with survival $(11,19)$. Limitations notably exist in all previous studies, including a small sample size in the former and the majority of SCEC patients being non-metastatic. The present research shares the limitation of other SEER-data-based studies in that its interpretation is restricted. As we also failed to investigate tumour invasion, distant metastases, as well as lymph node metastases of $27.2-52.2 \%$ of patients, there were $138(32.3 \%)$ and $139(32.6 \%)$ patients with unknown 7th and 6th TNM stage. However, both the AJCC and SEER categorizations of parameters only incorporate tumourrelated variables, and the clinical characteristics of patients 

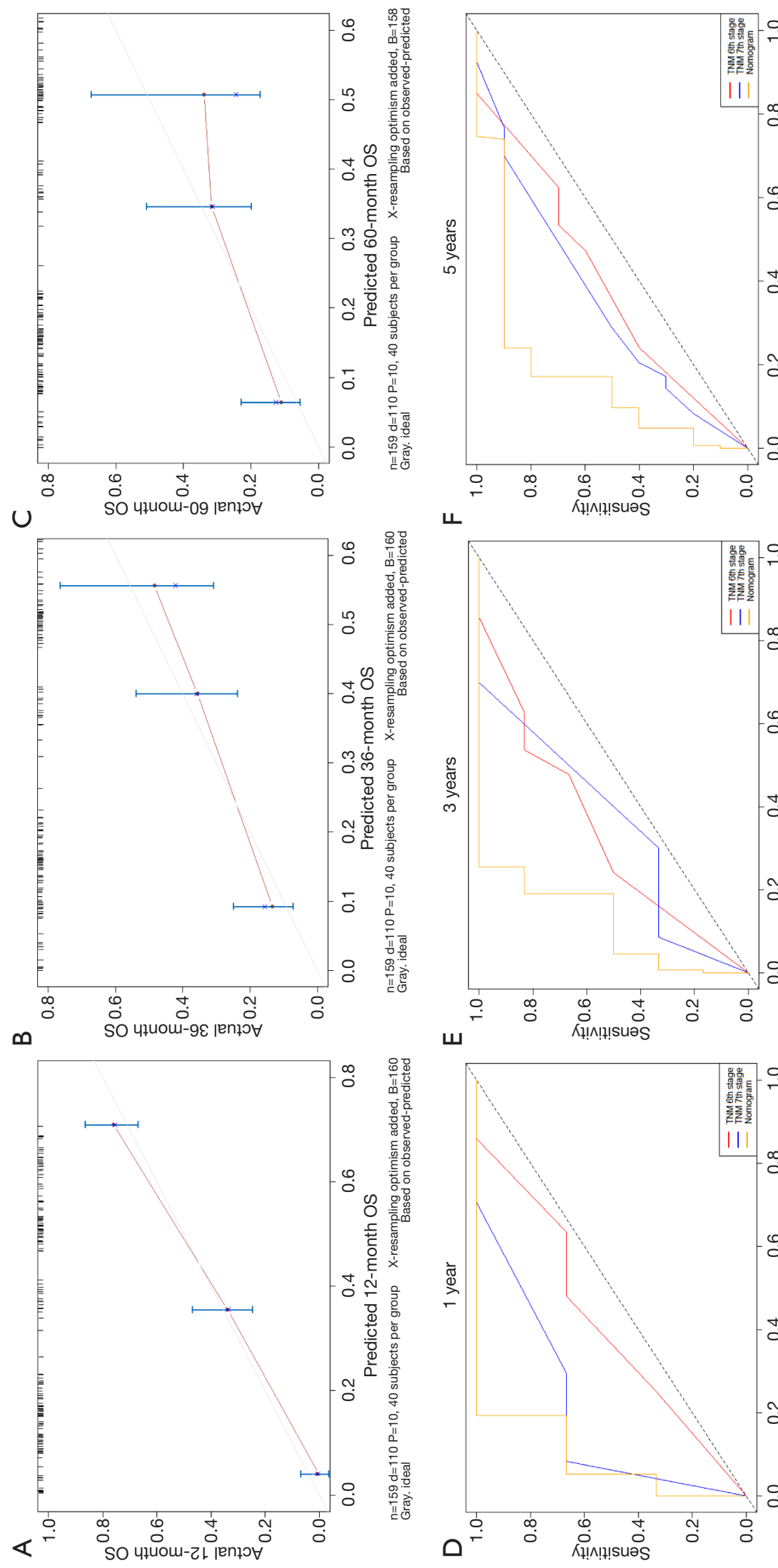$$
\text { ш }
$$
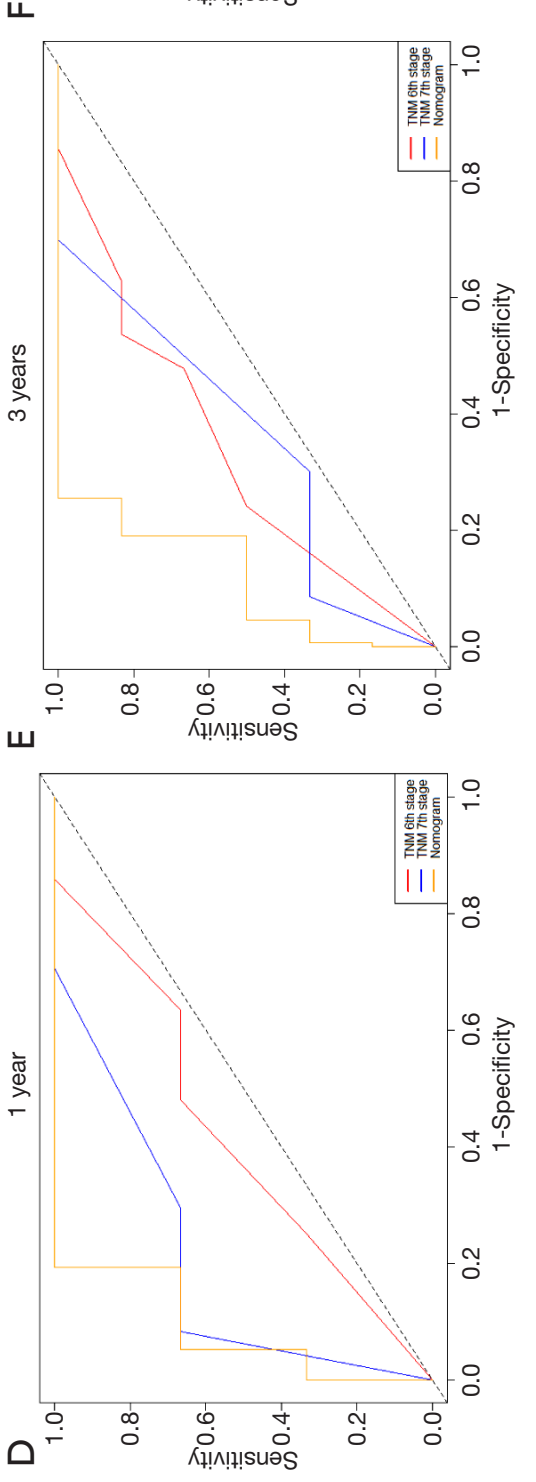

I<smiles>[CH]1CC1</smiles>

है

$\vartheta$

 
and data on previous therapies is not taken into account, resulting in an inaccurate estimation of OS as several studies in other cancer types have revealed $(15,16,20,21)$. Future studies are required to establish a more precise and individual staging system specified for SCEC.

In the current study, besides the SEER stage, we showed that age, male gender, tumor location in the upper-third of the esophagus, and insufficient or no radiotherapy, chemotherapy, and/or surgery were closely linked to mortality in SCEC patients. Our data for age $(9,13,18,22)$, male gender $(9,18,22)$, and primary tumour site $(9,22)$ as prognostic factors accords with previously published literature on SCEC. Zhang and colleagues (23) built a competing-risk model to assess the cause-specific survival (CSS) in patients with esophageal NEC which demonstrated that primary tumour site, distant metastases, and clinical treatment were closely linked to CSS, which supports our results.

Zhang's study also concluded surgery was a protective factor that lowered the possibility of cause-specific mortality in SCEC patients by $76 \%$, followed by radiotherapy and chemotherapy, whereas our results indicated that the death risk of patients treated with chemotherapy decreased by $62 \%(\mathrm{HR}=0.38,95 \% \mathrm{CI}, 0.30-0.49)$, followed by radiotherapy ( $\mathrm{HR}=0.71,95 \% \mathrm{CI}, 0.57-0.89$ ), and surgery (HR $=0.75,95 \%$ CI, 0.62-0.91). This difference may be because Zhang's nomogram enrolled 162 esophageal NEC patients from 1998 to 2004 making the data relatively old, and may have included patients with large cell carcinomas. Moreover, Zhang's nomogram did not include any staging system, which is a major limitation acknowledged by the authors in their paper and its editorial letter (24). In addition, they did not verify their nomogram externally. An optimal treatment strategy has not been provided in national guidelines for SCEC due to a lack of sufficient data. SCEC is widely acknowledged as a complicated disease, and metastatic disease has occurred in $40-60 \%$ of patients at the time of diagnosis (5). While chemotherapy was regarded as the foundation of multidisciplinary as well as local treatment for SCEC, the recurrence rate has remained high and the prognosis far from ideal $(5,11,25)$, and while surgery continues to play a predominant role, its use is limited to early stage and/or resectable SCEC patients $(6,19,26,27)$. In the current study, we enrolled 427 SCEC patients from the SEER database and $49.4 \%$ patients were distant SEER stage, which is a figure consistent with the literature and may indicate our results are close to the real world. On this basis, we propose chemotherapy-based systemic therapy for the local treatment of SCEC.

However, there are several limitations to this study. Above all, the information relevant to tumour site, TNM and VALSG stage, lifestyle, performance status, pure/mixed SCEC, surgical margins, and other detailed clinical information could not be ascertained from SEER, which restricted the reliability as well as precision of our prognostic analyses. Secondly, our study developed a nomogram to estimate individualized OS, which did not take competing risks into consideration, and more investigation into cause-specific mortality in SCEC patients is warranted. Last but not least, the retrospective nature and limited sample size of our analysis inevitably produced substantial bias, and prospective and multicentre studies are required to validate these factors.

\section{Conclusions}

Despite its limitations, as well as the rarity of SCEC, the nomogram in our research was developed and verified on the basis of a large population of SCEC patients and on the basis of combining non-anatomic factors with anatomic ones, incorporating both clinical characteristics and therapy methods. While the estimations of the nonogram were relatively accurate when predicting the prognosis of SCEC patients, more research based on larger-scale samples is required to validate its applicability and update our findings.

\section{Acknowledgments}

We thank the American Journal Experts (https://www.aje. $\mathrm{com} /$ ) for editing this manuscript.

Funding: The study was supported by the Fund for Young Doctors from the Shanghai Anticancer Association (Grant No. SACA-CY19C12).

\section{Footnote}

Reporting Checklist: The authors have completed the TRIPOD reporting checklist. Available at https://dx.doi. org/10.21037/atm-21-3900

Conflicts of Interest: All authors have completed the ICMJE uniform disclosure form (available at https://dx.doi. org/10.21037/atm-21-3900). The authors have no conflicts of interest to declare.

Ethical Statement: The authors are accountable for all 
aspects of the work in ensuring that questions related to the accuracy or integrity of any part of the work are appropriately investigated and resolved. This retrospective study was approved by the institutional review board and independent ethics committee of FUSCC. The study was conducted in accordance with the Declaration of Helsinki (as revised in 2013).

Open Access Statement: This is an Open Access article distributed in accordance with the Creative Commons Attribution-NonCommercial-NoDerivs 4.0 International License (CC BY-NC-ND 4.0), which permits the noncommercial replication and distribution of the article with the strict proviso that no changes or edits are made and the original work is properly cited (including links to both the formal publication through the relevant DOI and the license). See: https://creativecommons.org/licenses/by-nc-nd/4.0/.

\section{References}

1. van der Veen A, Seesing MFJ, Wijnhoven BPL, et al. Management of resectable esophageal and gastric (mixed adeno)neuroendocrine carcinoma: A nationwide cohort study. Eur J Surg Oncol 2018;44:1955-62.

2. Honma $\mathrm{Y}$, Nagashima K, Hirano H, et al. Clinical outcomes of locally advanced esophageal neuroendocrine carcinoma treated with chemoradiotherapy. Cancer Med 2020;9:595-604.

3. Garcia-Carbonero R, Sorbye H, Baudin E, et al. ENETS Consensus Guidelines for High-Grade Gastroenteropancreatic Neuroendocrine Tumors and Neuroendocrine Carcinomas. Neuroendocrinology 2016;103:186-94.

4. Yuan G, Zhan C, Zhu D, et al. Population-based analysis of esophageal large cell neuroendocrine carcinoma between 2004 and 2015. J Thorac Dis 2019;11:5480-8.

5. Lv J, Liang J, Wang J, et al. Primary small cell carcinoma of the esophagus. J Thorac Oncol 2008;3:1460-5.

6. Purwar P, Jiwnani S, Karimundackal G, et al. Management of esophageal small cell carcinoma. Ann Thorac Surg 2015;99:1488.

7. Liu D, Xu X, Wen J, et al. Integrated Genome-Wide Analysis of Gene Expression and DNA Copy Number Variations Highlights Stem Cell-Related Pathways in Small Cell Esophageal Carcinoma. Stem Cells Int 2018;2018:3481783.

8. Zhang Z, Xiao H, Xie F, et al. High-incidence of PTEN mutations in Chinese patients with primary small cell carcinoma of the esophagus. BMC Cancer 2014;14:19.

9. Meng MB, Zaorsky NG, Jiang C, et al. Radiotherapy and chemotherapy are associated with improved outcomes over surgery and chemotherapy in the management of limitedstage small cell esophageal carcinoma. Radiother Oncol 2013;106:317-22.

10. Zhu Y, Qiu B, Liu H, et al. Primary small cell carcinoma of the esophagus: review of 64 cases from a single institution. Dis Esophagus 2014;27:152-8.

11. Ding J, Ji J, Zhu W, et al. A retrospective study of different treatments of limited-stage small-cell esophageal carcinoma and associated prognostic factor analysis. Dis Esophagus 2013;26:696-702.

12. Verma V, Sleightholm RL, Fang P, et al. National Cancer Database report of nonmetastatic esophageal small cell carcinoma. Cancer Med 2018;7:6365-73.

13. Al Mansoor S, Ziske C, Schmidt-Wolf IG. Primary small cell carcinoma of the esophagus: patient data metaanalysis and review of the literature. Ger Med Sci 2013;11:Doc12.

14. Balachandran VP, Gonen M, Smith JJ, et al. Nomograms in oncology: more than meets the eye. Lancet Oncol 2015;16:e173-80.

15. Liang $W$, Zhang L, Jiang G, et al. Development and validation of a nomogram for predicting survival in patients with resected non-small-cell lung cancer. J Clin Oncol 2015;33:861-9.

16. Wang Y, Li J, Xia Y, et al. Prognostic nomogram for intrahepatic cholangiocarcinoma after partial hepatectomy. J Clin Oncol 2013;31:1188-95.

17. Wen J, Liu D, Xu X, et al. Nomograms for predicting survival outcomes in patients with primary tracheal tumors: a large population-based analysis. Cancer Manag Res 2018;10:6843-56.

18. Kukar M, Groman A, Malhotra U, et al. Small cell carcinoma of the esophagus: a SEER database analysis. Ann Surg Oncol 2013;20:4239-44.

19. Chen WW, Wang F, Chen S, et al. Detailed analysis of prognostic factors in primary esophageal small cell carcinoma. Ann Thorac Surg 2014;97:1975-81.

20. Dong F, Shen Y, Gao F, et al. Nomograms to Predict Individual Prognosis of Patients with Primary Small Cell Carcinoma of the Bladder. J Cancer 2018;9:1152-64.

21. Qin BD, Jiao XD, Zang YS. Primary pulmonary leiomyosarcoma: A population-based study. Lung Cancer 2018;116:67-72.

22. Wang HH, Zaorsky NG, Meng MB, et al. Multimodality therapy is recommended for limited-stage combined small cell esophageal carcinoma. Onco Targets Ther 
2015;8:437-44.

23. Zhang G, Wu B, Wang X, et al. A competing-risks nomogram and recursive partitioning analysis for cause-specific mortality in patients with esophageal neuroendocrine carcinoma. Dis Esophagus

2019;32:doy129. Erratum in: Dis Esophagus 2019;32(6): PMID: 30715226.

24. Zhang G, Li J. Reply to the Editor: how to treat esophageal neuroendocrine carcinoma with proper stratification? Dis Esophagus 2019;32:doz036.

25. Casas F, Ferrer F, Farrús B, et al. Primary small cell

Cite this article as: Liu D, Wen J, Chen J, Fan M, Zhang Z. Nomogram for the prediction of individualized overall survival of patients diagnosed with small cell esophageal carcinoma. Ann Transl Med 2021;9(16):1344. doi: 10.21037/atm-21-3900 carcinoma of the esophagus: a review of the literature with emphasis on therapy and prognosis. Cancer 1997;80:1366-72.

26. Hou X, Wei JC, Wu JX, et al. Multidisciplinary modalities achieve encouraging long-term survival in resectable limited-disease esophageal small cell carcinoma. PLoS One 2013;8:e69259.

27. Lu XJ, Luo JD, Ling Y, et al. Management of small cell carcinoma of esophagus in China. J Gastrointest Surg 2013;17:1181-7. 


\section{Supplementary}

Table S1 Detailed scores of all predictors in the nomogram

\begin{tabular}{|c|c|}
\hline Variables & Nomogram points \\
\hline \multicolumn{2}{|l|}{ Age } \\
\hline 20 & 0 \\
\hline 25 & 7 \\
\hline 30 & 14 \\
\hline 35 & 21 \\
\hline 40 & 29 \\
\hline 45 & 36 \\
\hline 50 & 43 \\
\hline 55 & 50 \\
\hline 60 & 57 \\
\hline 65 & 64 \\
\hline 70 & 71 \\
\hline 75 & 78 \\
\hline 80 & 86 \\
\hline 85 & 93 \\
\hline 90 & 100 \\
\hline \multicolumn{2}{|l|}{ Gender } \\
\hline Female & 0 \\
\hline Male & 12 \\
\hline \multicolumn{2}{|l|}{ Location } \\
\hline Low & 0 \\
\hline Overlap & 12 \\
\hline Middle & 13 \\
\hline Upper & 14 \\
\hline \multicolumn{2}{|l|}{ Stage } \\
\hline Localized & 0 \\
\hline Regional & 28 \\
\hline Distant & 86 \\
\hline Unstaged & 35 \\
\hline \multicolumn{2}{|l|}{ Surgery } \\
\hline Yes & 0 \\
\hline No & 5 \\
\hline \multicolumn{2}{|c|}{ Radiotherapy } \\
\hline Yes & 0 \\
\hline No & 27 \\
\hline \multicolumn{2}{|c|}{ Chemotherapy } \\
\hline Yes & 0 \\
\hline No & 76 \\
\hline
\end{tabular}

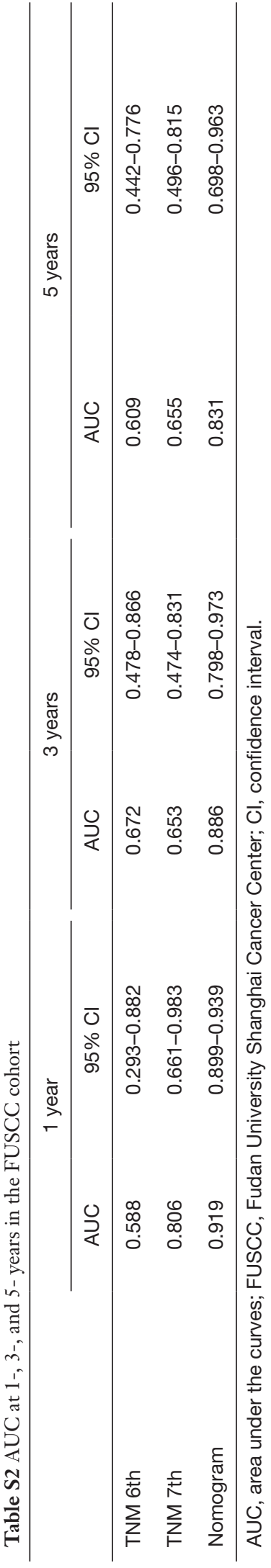

(c) Annals of Translational Medicine. All rights reserved. 